Claremont Colleges

Scholarship@Claremont

WM Keck Science Faculty Papers

W.M. Keck Science Department

$12-1-1995$

\title{
Complex Dynamics and Multistability in a Damped Harmonic Oscillator with Delayed Negative Feedback
}

Sue Ann Campbell

University of Waterloo

Jacques Bélair

University of Montreal

Toru Ohira

John Milton

Claremont McKenna College; Pitzer College; Scripps College

\section{Recommended Citation}

Campbell, Sue Ann, Jacques Belair, Toru Ohira, and John Milton. "Complex Dynamics and Multistability in a Damped Harmonic Oscillator with Delayed Negative Feedback." Chaos 5.4 (1995): 640-645. DOI: 10.1063/1.166134

This Article is brought to you for free and open access by the W.M. Keck Science Department at Scholarship @ Claremont. It has been accepted for inclusion in WM Keck Science Faculty Papers by an authorized administrator of Scholarship @ Claremont. For more information, please contact scholarship@cuc.claremont.edu. 


\title{
Complex dynamics and multistability in a damped harmonic oscillator with delayed negative feedback
}

\author{
Sue Ann Campbell \\ Centre for Nonlinear Dynamics in Physiology and Medicine, McGill University, Montréal, Canada and \\ Department of Applied Mathematics, University of Waterloo, Waterloo, Canada and Centre de Recherches \\ Mathématiques, Université de Montréal, Montréal, Canada \\ Jacques Bélair \\ Centre for Nonlinear Dynamics in Physiology and Medicine, McGill University, Montréal, Canada and \\ Centre de Recherches Mathématiques and Départment de Mathématiques et de Statistique, Université de \\ Montréal, Montréal, Canada \\ Toru Ohira \\ Sony Computer Science Laboratory, Inc., Tokyo, Japan \\ John Milton a) \\ Department of Neurology and Committee on Neurobiology, The University of Chicago Hospitals, MC2030, \\ 5841 South Maryland, Chicago, Illinois 60637 and Centre for Nonlinear Dynamics in Physiology and \\ Medicine, McGill University, Montréal, Canada
}

(Received 13 June 1994; accepted for publication 23 May 1995)

A center manifold reduction and numerical calculations are used to demonstrate the presence of limit cycles, two-tori, and multistability in the damped harmonic oscillator with delayed negative feedback. This model is the prototype of a mechanical system operating with delayed feedback. Complex dynamics are thus seen to arise in very plausible and commonly occurring mechanical and neuromechanical feedback systems. (C) 1995 American Institute of Physics.

\section{INTRODUCTION}

Time delays are intrinsic and important features of many physical and biological control systems. ${ }^{1-4}$ The time delays most commonly occur as a consequence of finite conduction and production times. Here we study the dynamics of differential delay equations that arise in the delayed feedback control of mechanical systems. ${ }^{1,5-7}$ Considerations of Newton's laws of motion lead to second-order delay-differential equations of which the prototype is the damped harmonic oscillator with a delayed restoring force (Fig. 1),

$$
\ddot{x}+b \dot{x}+a x=f\left(x_{\tau}\right)
$$

where $a, b$ are constants, $\tau$ is the time delay, $x, x_{\tau}$ are the displacement at times $t, t-\tau$, respectively, and the function, $f$, describes the feedback. Equation (1) also arises as an approximation to second-order systems acting under the influence of feedback, which is a function of displacement and velocity: a function $g(x, \dot{x})$ is replaced by $g\{x(t),[x(t)-x(t$ $-\tau)] / \tau\} \equiv p[x(t), x(t-\tau)]$. Applications of (1) include the neuromuscular regulation of movement and posture, ${ }^{4}$ acousto-optical bistability, ${ }^{8}$ metal cutting, ${ }^{9,10}$ the cascade control of fluid level devices ${ }^{11,12}$ and the electronically "clamped" pupil light reflex. ${ }^{13,14}$

Complex oscillations, including chaos and two-tori, have been shown to occur in (1) when $f$ is a nonmonotone function. ${ }^{11,12}$ Recently, it has been demonstrated analytically by performing a center manifold reduction that two-tori can also be produced by (1) when $f$ is simple monotone negative feedback,${ }^{15}$ i.e.

\footnotetext{
${ }^{\text {a) }}$ To whom, correspondence should be addressed.
}

$$
f\left(x_{\tau}\right)=\frac{\theta^{n}}{\theta^{n}+x_{\tau}^{n}}+k,
$$

where $n, \theta$, and $k$ are positive constants. Negative feedback control arises far more commonly in practical applications than nonmonotone types of feedback do. Thus, the observation that complex dynamics can arise from generic secondorder differential delay equations with monotone negative feedback is of great interest.

Here we integrate (1) with negative feedback, i.e. (2), numerically and determine the nature of the bifurcations of the two-tori. The local stability analysis of (1) is outlined in Sec. II and the center manifold reduction is discussed in Sec. III. Numerical studies demonstrate that in certain parameter ranges there is a coexistence of stable two-tori and two qualitatively different limit cycles. In Sec. V it is shown that this multistability can be explained by the inclusion of higherorder terms (i.e., fifth order) into the center manifold analysis.

\section{LOCAL STABILITY ANALYSIS}

Here we briefly discuss the local stability analysis of (1). More details can be found in Refs. 7 and 15-19.

The steady-state value of $x, x^{*}$, is obtained as a solution of

$$
a x^{*}=f\left(x^{*}\right),
$$

and linearization of (1) about $x^{*}$ leads to

$$
\ddot{u}+b \dot{u}+a u=d u_{\tau},
$$

where $u=x-x^{*}$ and $d=f^{\prime}\left(x^{*}\right)$. We make the usual ansatz $u=\exp (\lambda t)$, where $\lambda$ is a complex number, and obtain the characteristic equation, 


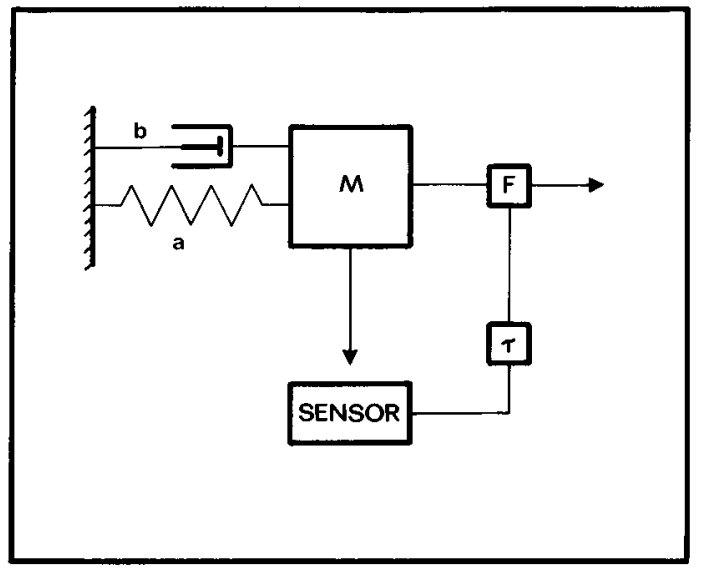

FIG. 1. Schematic diagram of a damped harmonic oscillator with a delayed restoring force.

$$
\lambda^{2}+b \lambda+a-d \exp (-\lambda \tau)=0 .
$$

Stability changes of $x^{*}$ can only occur for $\lambda=i \omega$ : substituting the latter into (3) and rearranging, we obtain

$$
\frac{a-\omega^{2}}{d}+i\left(\frac{\omega b}{d}\right)=\exp (-i \omega \tau) \text {. }
$$

Equation (4) can be solved graphically (Fig. 2): the right-hand side of (4) describes the unit circle in the complex plane. We only consider systems that are stable for small $\tau$, i.e. $a>0$, and $d<0$ for negative feedback. Thus, the left-hand side of (4) starts at the point $a / d<0$ when $\omega=0$ and as $\omega$ increases describes a parabola that traverses the complex plane from left to right. The solutions of (4), denoted $\omega_{+}$, are the positive solutions (in $\omega$ ) of the equation

$$
\omega^{4}+\left(b^{2}-2 a\right) \omega^{2}+\left(a^{2}-d^{2}\right)=0 .
$$

The values of the delay, $\tau_{j,+}$, at which a potential change in stability occurs must have one of the values

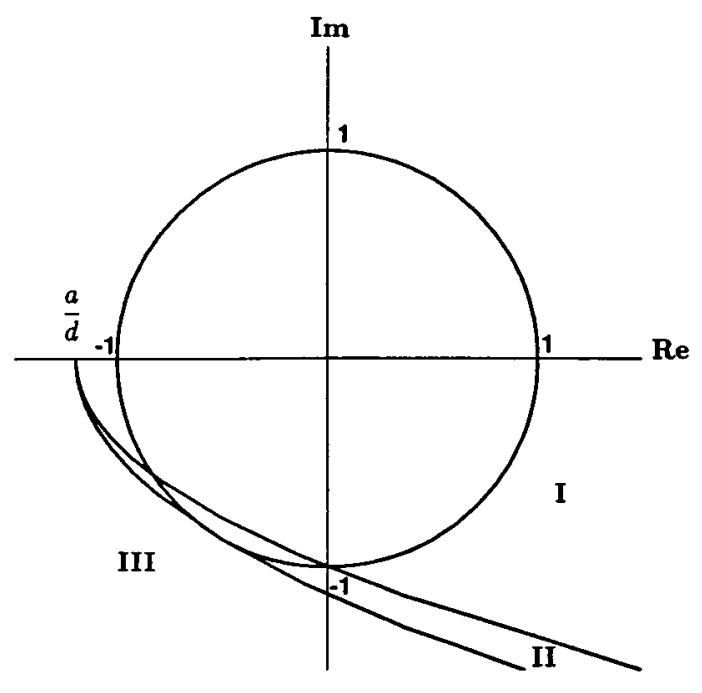

FIG. 2. Graphical method of the solution of Eq. (4).
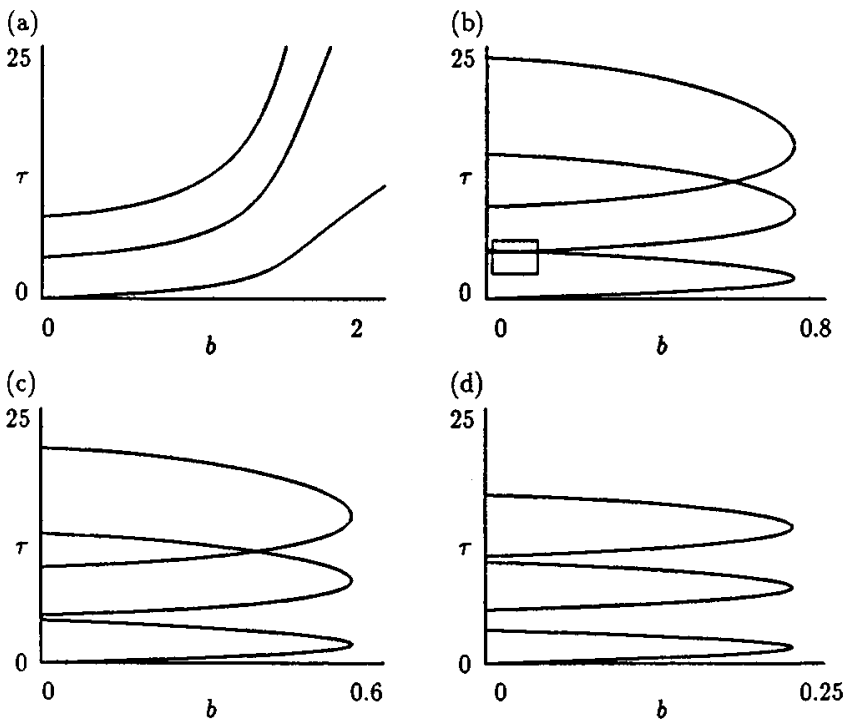

FIG. 3. Stability diagrams in the $(b, \tau)$ plane for (a) $d=-1.05(n=3.5)$, (b) $d=-0.63(n=2.1)$, (c) $d=-0.525(n=1.75)$, and (d) $d=-0.225(n=0.75)$. In all cases $a=1, c=1, k=\frac{1}{3}$, and $\theta=\frac{5}{6}$. Parameter values for which the equilibrium is stable are to the right of the solid lines, i.e. toward larger $b$. The vertical scale is the same for all graphs, but note differences in horizontal scale.

$$
\begin{gathered}
\tau_{j,+}=\frac{1}{\omega_{+}}\left[\tan ^{-1}\left(\frac{\omega_{+} b}{\omega_{+}^{2}-a}\right)+2 j \pi\right], \\
j=0, \pm 1, \pm 2, \ldots .
\end{gathered}
$$

The ratio $a / d$ can be identified with the gain, $G$, of the feedback, i.e.

$$
G=\left(\frac{a}{d}\right)^{-1},
$$

and plays an important role in the local stability analysis. If $a / d>-1$, then it is always possible to destabilize the steady state by increasing $\tau$. On the other hand, if $a / d<-1$, then increases in $\tau$ may or may not result in destabilization. In particular, there will be two positive roots of (5) if $\sqrt{2 a-2 \sqrt{a^{2}-d^{2}}} \geqslant b>0$ and no positive roots otherwise. Thus it is necessary to estimate the magnitude of $a / d$.

Two-dimensional tori arise from mode interactions between two pairs of complex eigenvalues, the real parts of which become positive simultaneously. Since each solution, $\omega_{+}$, of (5) is associated with a pair of complex eigenvalues, this condition for the existence of a two-tori requires that there be two solutions $\omega_{+}$of (5), and hence that $a / d<-1$.

Under this last restriction, the stability of the steady state of (1) depends on the values of the damping coefficient, $b$, and the time delay $\tau$ (Figs. 2 and 3). When

$$
\frac{d}{\sqrt{a}} \geqslant b>0, \quad \text { Region } I,
$$

it can be shown that there is only a single change of stability as $\tau$ increases [Fig. 3(a)]. This follows from the fact (shown 
in Ref. 16) that no tangency of the roots can take place on the imaginary axis, and that stability is indeed lost when $\lambda=i \omega$. As $b$ is increased further to

$$
\sqrt{2 a-2 \sqrt{a^{2}-d^{2}}} \geqslant b>\frac{d}{\sqrt{a}}, \text { Region II, }
$$

there can be a switching of stability as $\tau$ increases. ${ }^{15}$ This behavior gives rise to the characteristic "Christmas tree" stability diagram. ${ }^{17}$ Depending on the exact values of the parameters, a variety of Christmas tree stability diagrams can arise, some of which have one or more disjoint branches [Figs. 3(b)-3(d)]. Finally, for

$$
b>\sqrt{2 a-2 \sqrt{a^{2}-d^{2}}}, \text { Region III, }
$$

the steady state of (1) is stable for all $\tau$.

It should be noted that if the control mechanism is overdamped, then $b>\sqrt{4 a}$ and hence the stationary solution $x^{*}$ is stable for all $\tau$ when $a / d<-1$. Thus, if limit cycles and two-tori are to be generated by (1), then the control mechanism must be underdamped, i.e. $b<\sqrt{4 a}$.

\section{CENTER MANIFOLD REDUCTION}

When one branch of the stability boundary shown in Fig. 3 is crossed, the steady state destabilizes as a pair of complex conjugate eigenvalues passes through the imaginary axis. This is the signature of a Hopf bifurcation, and we might expect a stable limit cycle to appear. This Hopf bifurcation can be either supercritical or subcritical, and thus the limit cycle may be stable or unstable, depending on the values of the parameters. ${ }^{15}$ This situation should be contrasted to that of a first-order differential equation with delayed negative feedback [with function (2)] in which the Hopf bifurcation is always supercritical. ${ }^{13}$

Here, we concentrate on the situation where two branches of the stability boundary intersect [see the box in Fig. 3(b)]. At such points there are two values of $\omega$ satisfying (4) for the same value of $\tau$ and $b$ and thus, correspondingly, there are two pairs of pure imaginary eigenvalues $\pm i \omega_{1}$, $\pm i \omega_{2}$. These points are called double Hopf bifurcation points. The behavior of a system of ordinary or delay differential equations having eigenvalues with zero real parts can be determined by using the techniques of center manifold reduction $^{20,21}$ and normal form analysis. ${ }^{22}$ In our case there are four such eigenvalues, and thus it is possible to construct a four-dimensional manifold. The flow on this manifold represents the long term behavior of the full system in the neighborhood of the bifurcation point. This algebraically daunting task (for a discussion see Ref. 15) has recently been facilitated with the use of symbolic manipulation computer software. ${ }^{23}$

The emphasis in this study is on the dynamics of (1) in the neighborhood of the double Hopf bifurcation point (i.e., along the line $a \rightarrow b \rightarrow c \rightarrow d$ in Fig. 4). In this section we briefly summarize the results of the center manifold reduction carried out to terms of third order. A detailed derivation and analysis can be found in Ref. 15.

At the first intersection point in the bifurcation set of Fig. 3(b) (see the box), we find that $d=-0.63$ and the coor-

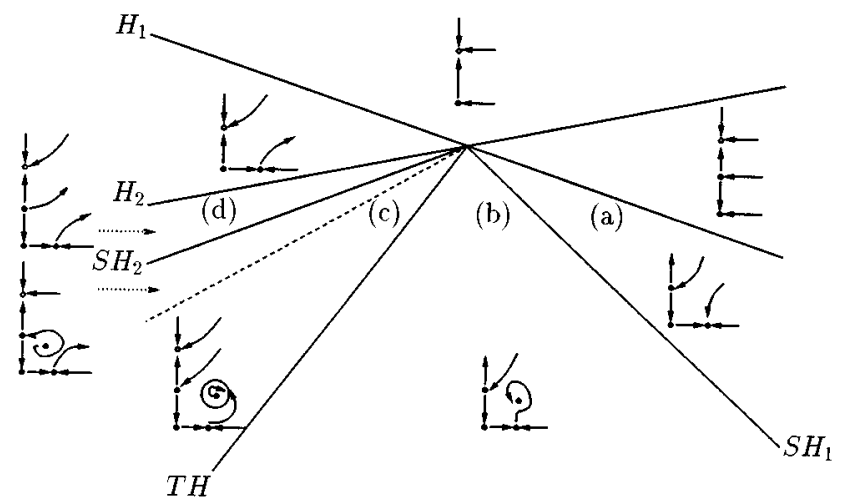

FIG. 4. Bifurcation set near the first intersection point $(\tau=5.04, b=0.07)$ at $a=1, d=-0.63$ [the box in Fig. 3(b)]. Filled circles represent fixed points (periodic solutions) predicted by the theory, open circles represent fixed points not predicted by theory but observed numerically. The letters (a)-(d) indicate the parameter values for the simulations of Figs. 5 and 6 .

dinates of the intersection point are $b=0.070=b_{\text {int }}$ and $\tau=5.043=\tau_{\text {int }}$, which corresponds to $\omega_{1}=0.609=\omega_{1 \text { int }}$ on the first branch and $\omega_{2}=1.274=\omega_{2 \text { int }}$ on the second branch. At this point the flow on the center manifold is described by the following system of four ordinary differential equations (after a reduction to normal form and transformation to polar coordinates),

$$
\begin{aligned}
& \dot{r}_{1}=-0.0247 r_{1}^{3}-0.194 r_{1} r_{2}^{2}, \\
& \dot{r}_{2}=0.0437 r_{1}^{2} r_{2}+0.0068 r_{2}^{3}, \\
& \dot{\theta}_{1}=\omega_{1 \text { int }}, \\
& \dot{\theta}_{2}=\omega_{2 \text { int }} .
\end{aligned}
$$

For parameter values close to this double bifurcation point the dynamics of the system can be described by the unfolding

$$
\begin{aligned}
& r_{1}=\mu_{1} r_{1}-0.0247 r_{1}^{3}-0.194 r_{1} r_{2}^{2}, \\
& \dot{r}_{2}=\mu_{2} r_{2}+0.0437{ }_{1}^{2} r_{2}+0.0068 r_{2}^{3}, \\
& \dot{\theta}_{1}=\omega_{1 \text { int }}, \\
& \dot{\theta}_{2}=\omega_{2 \text { int }},
\end{aligned}
$$

where the parameters $\mu_{1}$ and $\mu_{2}$, respectively, represent directions in parameter space perpendicular to the second and first Hopf bifurcation curves of Fig. 3(b). Locally, we may represent these curves as straight lines and thus express $\mu_{1}$, $\mu_{2}$ in terms of $b, \tau$, viz.:

$$
\begin{aligned}
\mu_{1} & =\alpha_{1}\left(\tau-\tau_{\text {int }}\right)+\beta_{1}\left(b-b_{\text {int }}\right) \\
& =-0.470\left(\tau-\tau_{\text {int }}\right)-0.883\left(b-b_{\text {int }}\right), \\
\mu_{2} & =\alpha_{2}\left(\tau-\tau_{\text {int }}\right)+\beta_{2}\left(b-b_{\text {int }}\right) \\
& =0.470\left(\tau-\tau_{\text {int }}\right)-0.883\left(b-b_{\text {int }}\right) .
\end{aligned}
$$

Note that in (11) the equations for $r_{1}, r_{2}$ do not depend on the variables $\theta_{1}, \theta_{2}$. Thus, by employing the substitution (12) we need only consider the following two equations to completely determine the dynamics of the system: 
TABLE I. Solution correspondence.

\begin{tabular}{|c|c|}
\hline Solution in Eqs. (13) & Solution in (11) \\
\hline Fixed point at origin & Fixed point at origin \\
\hline Fixed point $\left(\hat{r}_{1}, 0\right)$ & Periodic solution with period $2 \pi / \omega_{\text {lint }}$ \\
\hline Fixed point $\left(0, \hat{r}_{2}\right)$ & Periodic solution with period $2 \pi / \omega_{2 \text { int }}$ \\
\hline Fixed point $\left(\hat{r}_{1}, \hat{r}_{2}\right)$ & $\begin{array}{c}\text { Two-torus (quasiperiodic solution with two } \\
\text { frequencies) }\end{array}$ \\
\hline Periodic solution & $\begin{array}{c}\text { Three-torus (quasiperiodic solution with three } \\
\text { frequencies) }\end{array}$ \\
\hline
\end{tabular}

$$
\begin{aligned}
\dot{r}_{1}= & (2.432-0.470 \tau-0.883 b) r_{1}-0.0247 r_{1}^{3} \\
& -0.194 r_{1} r_{2}^{2}, \\
\dot{r}_{2}= & (-2.308+0.470 \tau-0.883 b) r_{2}+0.0437 r_{1}^{2} r_{2} \\
& +0.0068 r_{2}^{3} .
\end{aligned}
$$

The correspondence between solutions of (11) and of (13) is shown in Table I. Equation (13) with general coefficients has been studied by Guckenheimer and Holmes. ${ }^{20}$ The parameter values considered here correspond to the Case VIa of Ref. 20. (Other cases can also be observed. ${ }^{15}$ )

The relationship between these solutions is summarized in Fig. 4, which shows the bifurcation set for (13) in $b, \tau$ parameter space. The intersection point of all the bifurcation lines represents the first intersection point of Fig. 3(b). The lines $H_{1}, H_{2}$ are the curves of loss of stability of Fig. 3(b) (which appear as straight lines at this scale). These are supercritical and subcritical Hopf bifurcations that give rise, respectively, to periodic solutions with frequencies $\omega_{\text {lint }}$ and $\omega_{2 \text { int }}$. The other lines indicate secondary Hopf bifurcations $\left(\mathrm{SH}_{1}, \mathrm{SH}_{2}\right)$, giving rise to quasiperiodic solutions with two frequencies and a tertiary Hopf bifurcation $(\mathrm{TH})$ giving rise to quasiperiodic solution with three frequencies. It is likely that this three-torus is destroyed via a homoclinic bifurcation. ${ }^{20}$ However, the exact location of this bifurcation cannot be computed from (13), we have thus represented it by a dashed line in Fig. 4 . The small pictures illustrate (in the $r_{1}, r_{2}$ plane) the existence and stability of solutions to (13) at parameter values in each region.

\section{MULTISTABILITY}

For comparison with the analysis of Sec. III, we performed numerical simulations of the original equation (1) using a fourth-order Runge-Kutta scheme adapted for delay equations. The result of each simulation is displayed in two formats: time histories $[x(t)$ vs $t]$ in Fig. 5 and pseudophase space $[\dot{x}(t)$ vs $x(t)$ vs $x(t-\tau)]$ in Fig. 6 . The simulations were performed at parameter values in a neighborhood of the double bifurcation point depicted in Fig. 4. Further, we fixed the value of $\tau=5.03$ and varied $b$ as our bifurcation parameter. The values used for $b$ are indicated by the sequence of letters in Fig. 4, which correspond to the labels in Figs. 5 and 6.

The first two simulations confirm our analysis. That is, at (a) there is a limit cycle with frequency $\approx \omega_{\text {lint }} / 2 \pi=0.097$,
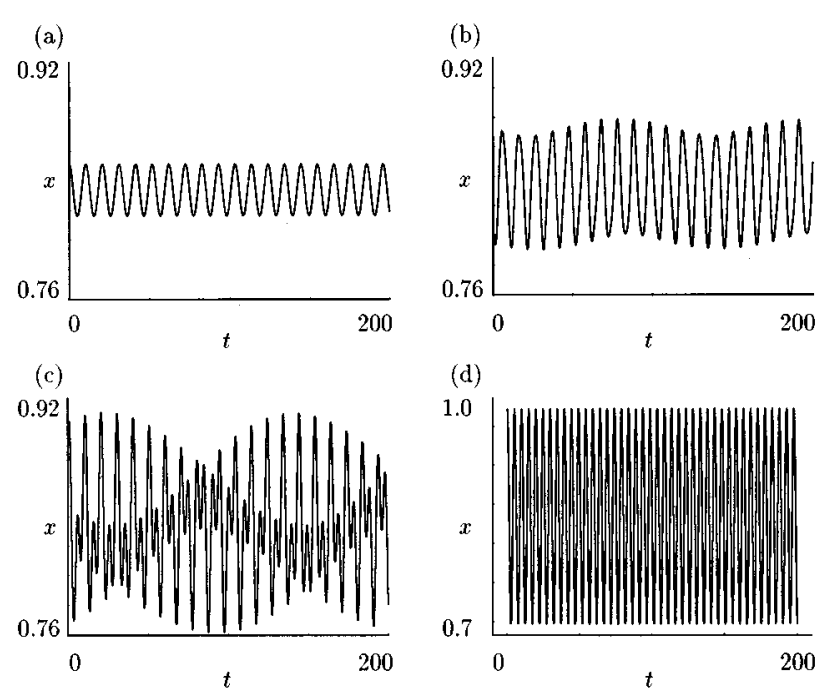

FIG. 5. Numerical simulation of (1) at $\tau=5.03$ for (a) $b=0.073$, (b) $b=0.071$, (c) $b=0.069$, and (d) $b=0.067$. Parameter values are the same as used in Fig. 3. Initial function $\phi=x_{2} \cos \left(\omega_{2} s\right)$ with $\omega_{2}=1.274, x_{2}=0.1$, and $s \in[-\tau, 0]$.

and as we cross the line $S H_{1}$ to (b), a two-torus appears. However, the other two simulations yield more surprising results.

On crossing the line $T H$ to (c), the analysis of Sec. III predicts that the two-torus should be unstable. However, we see a more complicated phase portrait. Although somewhat confusing, this phase portrait appears to represent a two- (a)

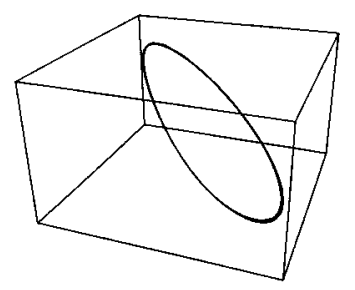

(c)

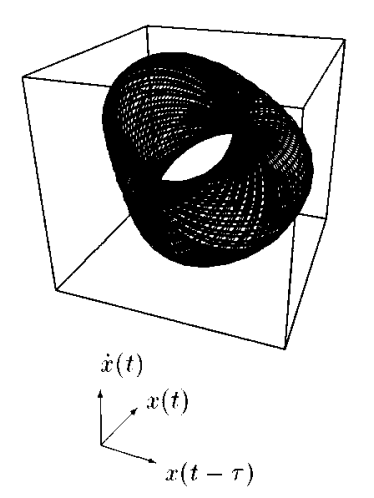

(b)

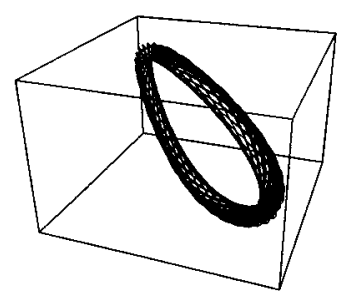

(d)

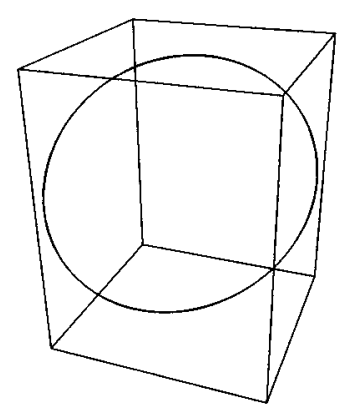

FIG. 6. Phase plane diagrams corresponding to the time histories of Fig. 5. Coordinates are $x(t-\tau)$ vs $x(t)$ vs $\dot{x}(t)$. 

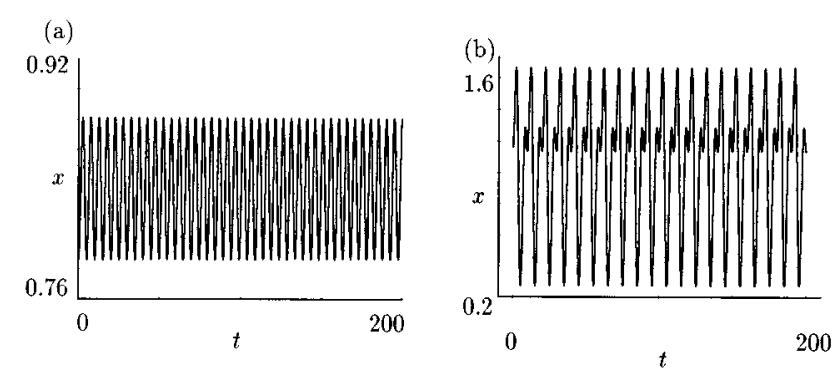

FIG. 7. Numerical simulations of (1) showing bistability. Parameter values are as in Fig. 6(c) $(\tau=5.03$ and $b=0.69)$ with different initial conditions $(\phi$ as in Fig. 5 with, respectively, $x_{2}=0.2$ and $x_{2}=0.5$ ). (a) Limit cycle, (b) period two limit cycle.

torus in which the amplitudes of the two frequencies are just about equal. [Figure 6(c) is comparable to a plot of $x(t)$ vs $\dot{x}(t) \quad$ vs $\quad x(t-\tau)$, where $\quad x(t)=0.8333+0.038 \sin \left(\omega_{1} t\right)$ $+0.038 \sin \left(\omega_{2} t\right)$, and the $\omega_{j}$ are the values of the frequencies of the two Hopf bifurcations $\left(\omega_{1}=0.60947\right.$ and $\left.\omega_{2}=1.27421\right)$.] We shall address the apparent contradiction between our analysis and this numerical result in the next section.

Finally, at (d) we see the appearance of a new periodic solution with frequency $\approx \omega_{2 \text { int }} / 2 \pi=0.202$ and with a considerably larger amplitude [note the difference in vertical scale between Figs. 5(a) $-5(\mathrm{c})$ and 5(d)]. The analysis in Sec. III predicts that no stable solution should exist at this point. We shall address the origin of this limit cycle, and how it raises the possibility of multistability at other parameter values, in the next section.

To explore this multistability we carried out numerical simulations with various initial conditions [i.e., $\phi(s)$ $=x_{1} \cos \left(\omega_{1} s\right)+y_{1} \sin \left(\omega_{1} s\right)+x_{2} \cos \left(\omega_{2} s\right)+y_{2} \sin \left(\omega_{2} s\right) \quad$ with $\omega_{1}=0.609, \omega_{2}=1.274, s \in[-\tau, 0]$, and various $\left.x_{j}, y_{j}\right]$ on a diverse set of parameters, including those used to generate Figs. 5 and 6. In particular, at the parameter values of Fig. 5(c), we found both the large-amplitude limit cycle [Fig. 7(a)] and an even larger-amplitude period two limit cycle [Fig. 7(b)] coexisting with the two-torus.

The limit cycle is observed numerically in other regions of parameter space as well: these are indicated in Fig. 4 by an open circle. Of particular interest is its existence in the region of stability of the fixed point. We note that the amplitude of the limit cycle in Fig. 7(a) is not particularly "large" in comparison with the amplitude of the coexistent twotorus. This would indicate that the limit cycle is near to annihilation in a saddle node bifurcation (see Sec. V). In fact, it is not observed in simulations carried out farther from the $\mathrm{H}_{2}$ Hopf bifurcation curve [at the parameter values of Figs. 5(a) and 5(b), for example].

The period two limit cycle was found to coexist with the other solution(s) for a large set of parameter values, almost everywhere that we carried out simulations.

\section{HIGHER-ORDER TERMS}

In order to understand the origin of the multistability discussed in Sec. IV, we included higher-order terms (up to the fifth order) into the center manifold reduction of (1) near its stationary solution. This yields the following system:

$$
\begin{aligned}
\dot{r}_{1}= & (2.432-0.470 \tau-0.883 b) r_{1}-0.0247 r_{1}^{3} \\
& -0.194 r_{1} r_{2}^{2}-0.1388 r_{1}^{5}, \\
\dot{r}_{2}= & (-2.308+0.470 \tau-0.883 b) r_{2}+.0437 r_{1}^{2} r_{2} \\
& +0.0068 r_{2}^{3}+0.1370 r_{1}^{2} r_{2}^{3}-0.0618 r_{2}^{5} .
\end{aligned}
$$

Consider first the effect due to the coefficient of the $r_{2}^{5}$ term in (14). Since it is negative while the term $r_{2}^{3}$ has a positive coefficient that is smaller in magnitude, one expects to find a region in parameter space where two fixed points of the form $\left(0, \hat{r}_{2}\right)$ of $(14)$ exist. Thus, we would expect to observe two limit cycles in this region for the original system: a small-amplitude, unstable one (created by the Hopf bifurcation $\mathrm{H}_{2}$ ) and a large-amplitude, stable one. These two cycles annihilate each other in a saddle-node bifurcation. Note that the large-amplitude stable cycle exists on both sides of the $\mathrm{H}_{2}$ Hopf bifurcation curve. More details on this degenerate (codimension 2) Hopf bifurcation can be found in Ref. 24.

In principle, (14) can also be used to estimate the location of the homoclinic bifurcation and hence to determine the stability of the three-torus referred to previously. This mainly numerical (albeit careful) work is left for a future investigation. However, numerical simulations performed on the normal form (14) itself, indicate that this three-torus should be stable.

We can further use (14) to refine the bifurcation curves of Fig. 4. The main result is that the three-torus is predicted to occur in some "curved wedge" between two parabolas that emanate from the double Hopf point. Since some of the coefficients of the third-order terms of the normal form are quite small, this refined wedge agrees with the previous one only when the $\mu_{j}$ are also small. This indicates that the thirdorder analysis is only valid for parameter values very close to the double Hopf point. Our simulations were not within this small region. Thus, not all of our numerical results can be predicted by the third-order analysis.

\section{DISCUSSION}

We have shown that limit cycles, two-tori, multistability, and other more complex dynamics can be generated by a generic second-order differential-delay equation with monotone negative feedback. Equations of this form arise in considerations of mechanical, or neuromechanical, systems operating under the influence of a delayed restoring force. The necessary conditions for these complex dynamics to occur, i.e. an underdamped control system $(b<\sqrt{4 a})$ with low gain $(G<-1)$ are easily satisfied by many mechanical systems. For example, we estimate from published data on a human postural control system (Fig. 49, p. 359, Ref. 25) that $b=4.1$ $\mathrm{s}^{-1}$ and $a=1044.5 \mathrm{~s}^{-2}$ (i.e., $b<\sqrt{a}$ ).

Typically numerical methods form the mainstay of techniques for exploring the behavior of nonlinear dynamical systems. Our studies emphasize the necessity of analytical work to guide these numerical experiments. Indeed, the ex- 
istence of two-tori for (1) can readily be overlooked if reference is not made to the center manifold reduction in choosing the appropriate parameter values. A case in point concerns the location of the predicted three-torus, which we have been unable to locate numerically.

An unusual feature of (1) is that limit cycles may arise via either a supercritical or a subcritical Hopf bifurcation. The subcritical Hopf bifurcation can give rise to higheramplitude limit cycles that coexist with the stable fixed point. This is an important phenomenon from a practical standpoint. It means that, depending on the initial conditions, one may observe oscillatory behavior in the "region of stability" of the equilibrium. The origin of the higher-amplitude limit cycles becomes clear when higher-order terms are added to the center manifold analysis. The presence of period two limit cycles in the system is not surprising, considering the possible homoclinic bifurcation described above. However, considering the amplitude of the period two limit cycles and their ubiquity in parameter space, we cannot rule out the possibility that they are linked to some other global bifurcation. Solutions similar to our period two limit cycles have been reported previously in a model of a delayed laser. ${ }^{26}$

The dynamic signature of a two-torus is the simultaneous presence of two frequencies in a time series. Time series of this type have indeed been observed for a number of physiological variables. Examples include finger position, ${ }^{27}$ pupil area in drowsy subjects, ${ }^{28}$ and insulin levels in healthy individuals. ${ }^{29}$ Since time delays are thought to be important features of the control mechanisms for these processes, it is tempting to speculate that these time series might reflect twotori generated by the control mechanism. However, it is not presently possible to eliminate the alternate possibility that the two frequencies represent the interaction of two distinct control mechanisms.

In first-order delay-differential equations, the appearance of complex dynamics is associated with nonmonotone types of feedback, such as "mixed" feedback. ${ }^{30}$ Although such feedback arises in specially constructed experimental paradigms, see, for example Ref. 31 , it is uncertain whether it is widespread naturally. One way in which nonmonotone feedback can arise is via the interaction of two or more feedback mechanisms. ${ }^{32}$ Here we have shown that complex dynamics arise in the generic second-order delay differential equations which arise in the delayed feedback control of mechanical systems. In contrast to first-order delay-differential equations, only monotone (negative) feedback is required. Thus complex dynamics can arise in very plausible and commonly occurring dynamical systems.

\section{ACKNOWLEDGMENTS}

We thank M. Delfour for useful comments. We acknowledge support from the National Institutes of Mental Health (USA), the National Sciences and Engineering Research Council (Canada), the Fonds pour la Formation de Chercheurs et l'Aide à la Recherche (Québec), and the North Atlantic Treaty Organization. Part of this work was performed while J.M. was at the Centre de Recherches Mathématiques at l'Université de Montréal.

${ }^{1}$ N. Minorsky, Nonlinear Oscillations (Van Nostrand, Princeton, NJ, 1962).

${ }^{2}$ V. B. Kolmanovskii and V. R. Nosov, Stability of Functional Differential Equations (Academic, New York, 1986).

${ }^{3}$ M. C. Mackey and J. G. Milton, Comments Theor. Biol. 1, 299 (1990).

${ }^{4}$ A. Beuter, J. Bélair, and C. Labrie, Bull. Math Biol. 55, 525 (1993).

${ }^{5}$ K. L. Bhatt and C. S. Hsu, J. Appl. Mech. 33, 113 (1966).

${ }^{6}$ C. S. Hsu and K. L. Bhatt, J. Appl. Mech. 33, 119 (1966).

${ }^{7}$ J. G. Milton and T. Ohira, Proc. World Cong. Nonlin. Anal. 4, 3085 (1995).

${ }^{8}$ R. Vallée, M. Dubois, M. Coté, and C. Delisle, Phys. Rev. A 36, 1327 (1987).

${ }^{9}$ D. W. Wu and C. R. Liu, ASME J. Eng. Ind. 17, 107 (1985).

${ }^{10}$ B. S. Berger, M. Rokni, and I. Minis, Q. Appl. Math. 51, 601 (1993).

${ }^{11}$ E. Boe and H-C. Chang, Chem. Eng. 44, 1281 (1989).

${ }^{12}$ E. Boe and H-C. Chang, Int. J. Bifurcation Chaos 1, 67 (1991).

${ }^{13}$ A. Longtin and J. G. Milton, Bull. Math. Biol. 51, 605 (1989).

${ }^{14}$ J. G. Milton and A. Longtin, Vision Res. 30, 515 (1990).

${ }^{15}$ S. A. Campbell, J. Bélair, J. Milton, and T. Ohira, J. Dyn. Diff. Eqs. 7, 213 (1995).

${ }^{16}$ K. L. Cooke and Z. Grossman, J. Math. Anal. Appl. 86, 592 (1982).

${ }^{17}$ F. G. Boese, J. Math. Anal. Appl. 140, 510 (1989).

${ }^{18}$ F. G. Boese and P. van den Driessche, Can. Appl. Math. Q. 2, 151 (1994).

${ }^{19}$ G. Stépán, Retarded Dynamical Systems, Pitman Research Notes in Mathematics (Longman, Essex, 1989), Vol. 210.

${ }^{20}$ J. Guckenheimer and P. J. Holmes, Nonlinear Oscillations, Dynamical Systems and Bifurcations of Vector Fields (Springer-Verlag, New York, 1983).

${ }^{21}$ J. K. Hale and S. M. Verduyn Lunel, Introduction to Functional Differential Equations (Springer-Verlag, New York, 1993).

${ }^{22}$ F. Takens, Pub. Math. IHES 43, 47 (1974).

${ }^{23}$ S. A. Campbell and J. Bélair, Can. Appl. Math. Q. (in press).

${ }^{24}$ M. Golubitsky and W. F. Langford, J. Diff. Eqs. 41, 525 (1981).

${ }^{25}$ L. Stark, Neurological Control Systems: Studies in Bioengineering (Plenum, New York, 1968).

${ }^{26}$ Y. Liu and J. Ohtsubo, Opt. Commun. 111, 317 (1994).

${ }^{27}$ A. Beuter, C. Labrie, and K. Vasilakos, Chaos 1, 279 (1991).

${ }^{28}$ R. E. Yoss, N. J. Moyer, and R. W. Hollenhorst, Neurology 20, 545 (1970).

${ }^{29}$ J. Sturis, K. S. Polonsky, E. T. Shapiro, J. D. Blackman, N. M. O’Meara, and E. van Cauter, Diabetologia 35, 681 (1992).

${ }^{30}$ M. C. Mackey and L. Glass, Science 197, 287 (1977).

${ }^{31}$ A. Longtin and J. G. Milton, Math. Biosci. 90, 183 (1988).

${ }^{32}$ L. Glass and C. P. Malta, J. Theor. Biol. 145, 217 (1990). 